\title{
Unités discursives de base et leur périphérie gauche dans LOCAS-F, un corpus oral multigenres annoté
}

\author{
Liesbeth Degand, Laurence J. Martin, Anne-Catherine Simon \\ Centre de recherche VALIBEL - Discours et Variation \\ Institut Langage \& Communication \\ \{liesbeth.degand, laurence.j.martin, anne-catherine.simon\}@uclouvain.be
}

\section{Introduction}

Cette contribution vise à présenter le corpus LOCAS-F (Louvain Corpus of Annotated Speech - French), développé à Louvain-la-Neuve (Belgique) depuis 2011 et annoté au niveau discursif. Au coeur de l'annotation se trouve l'unité discursive de base résultant de la corrélation entre unités prosodiques et unités syntaxiques (voir notamment Lacheret-Dujour et Victorri, 2002). Partant du postulat que la syntaxe et la prosodie fournissent chacune des signaux pour la délimitation d'unités dans le flux de parole, nous proposons que l'unité discursive soit définie par la coïncidence entre frontières syntaxique et prosodique. Le premier volet de cette contribution vise à présenter notre définition de ces unités, ainsi que notre méthode de segmentation, qui mêle une annotation syntaxique et une annotation prosodique, réalisées de manière totalement indépendante. Nous présentons ensuite l'ensemble du corpus LOCAS-F et ses différentes composantes, en nous attardant sur la distribution des unités discursives de base au sein des différents genres constituant notre corpus et justifions leur usage par rapport à la distribution des unités syntaxiques et des unités prosodiques seules. Nous poursuivons en effet l'hypothèse (en section 2) selon laquelle seule la combinaison de ces deux types d'unités permet de définir des unités pertinentes pour l'analyse du discours, remplissant une fonction cognitive dans la planification et l'interprétation de celuici. Enfin, nous présentons une étude exploitant le corpus annoté en nous concentrant sur les amorces des unités discursives. La périphérie gauche constitue le point d'ancrage du message et est le lieu, selon nous, où les locuteurs peuvent signaler la macro-structure de leur discours. Afin d'étudier les stratégies discursives à l'œuvre dans notre corpus, nous établissons dès lors une analyse distributionnelle des types formels observés et, sur un échantillon du corpus, nous comparons les différentes fonctions que ces éléments situés en périphérie gauche peuvent revêtir.

\section{Unités de segmentation du corpus}

\subsection{L'unité discursive de base}

La segmentation du discours en unités élémentaires n'est pas un sujet de préoccupation récent, et ne constitue pas une opération théorique neutre : le choix de segmentation est en effet indicatif de la manière d'appréhender la notion de discours. À ce sujet, deux conceptions différentes émergent de la littérature : la première conçoit le discours comme un objet hiérarchiquement structuré et donne la priorité à la représentation de la structure (sémantique) du discours, tandis que la seconde accorde davantage d'importance à l'aspect interactionnel du discours et à son déroulement dynamique.

La diversité des approches a donné lieu à différentes unités minimales constitutives du discours. Du côté des approches structurales, l'unité minimale correspond souvent à une unité syntaxique, la ponctuation ou la prosodie ne jouant pas de rôle prépondérant dans la détermination des unités de discours. Les unités émergentes de cette approche sont, parmi d'autres, les actes discursifs de Roulet et al. (2001) ou les unités de discours élémentaires de Asher et Lascarides (2003). Dans les approches interactionnelles (e.g. en analyse conversationnelle, voir Sacks, Schegloff et Jefferson 1974), on accorde une plus grande importance à l'intonation, les contours intonatifs pouvant constituer un locus pour le traitement cognitif de l'information précédente. Le contexte et l'organisation temporelle du discours jouent un rôle 
déterminant pour la définition des unités, appelées unités intonatives chez Chafe (1994), unités tonales chez Halliday (1994), ou encore unités de construction de tour chez Selting (2000).

En regard de ces deux types de modèles différents, nous pouvons reconnaître un double statut à ces segments constitutifs du discours, qui possèdent à la fois une face linguistique et une face discursive. La première se présente sous la forme d'une construction syntaxique dotée d'un contour prosodique, tandis que la seconde se présente sous la forme d'une unité informationnelle contextualisée. Selon Roulet (2002), il n'existe pas de frontière nette entre ces niveaux linguistique et discursif. Bien que conceptuellement différentes, ces deux approches peuvent, selon nous, être combinées dans une seule définition des segments constitutifs du discours. C'est pourquoi nous défendons ici la notion d'unité de base du discours (désormais BDU pour Basic Discourse Unit, Degand et Simon 2008, 2009a, 2009b, 2011), que nous définissons comme " the segments that speakers use to build a representation (interpretation) of the discourse, i.e. a kind of 'minimal discourse interpretation segments'» (Degand et Simon 2009a:4) et dont nous situons les propriétés fonctionnelles à l'intersection de cette double articulation linguistique et discursive. Les BDU sont effet des unités cognitives, que nous considérons à la fois comme des unités de traitement du discours (soit des structures achevées et interprétables) et des unités de production, construites en direct par un locuteur dans une situation plus ou moins interactive.

Afin de rendre compte du discours oral à la fois comme produit et comme processus, nous prenons pour point de départ l'analyse de surface du discours, à travers l'étude de sa structure syntaxique et des réalisations prosodiques. En effet, selon Halford (1996) et Selting (2000), ni la complétude syntaxique (Roulet 1991), ni la complétude prosodique (Chafe 1994) ne sont une condition suffisante pour la délimitation d'unités de base dans le flux de parole. Nous adoptons alors une méthode qui tient compte à la fois de la représentation statique de la structure du discours (unités syntaxiques et représentation sémantique) et de son déroulement dynamique (unités prosodiques offrant une présentation de surface du discours), en fondant notre segmentation sur les structures syntaxiques d'une part, et prosodiques d'autre part. Pour éviter tout problème de circularité, ces deux segmentations sont réalisées de manière totalement indépendante. La syntaxe et la prosodie fournissent ainsi chacune des signaux linguistiques pour la délimitation d'unités dans le discours, et la combinaison de ces marques permet de définir les unités à un niveau supérieur, qui correspond à l'organisation proprement discursive. Il existe différents types de BDU selon la manière dont syntaxe et prosodie sont conformées : nous détaillons ceux-ci au sein de la section 2.4 , après avoir brièvement présenté nos méthodes d'analyses syntaxique et prosodique dans les deux sections suivantes.

\subsection{Analyse syntaxique}

L'annotation syntaxique de notre corpus a été réalisée de manière manuelle, en double annotation, sur base des principes de la grammaire de dépendance (Blanche-Beneveniste et al. 1990), développée depuis les années 70 par le groupe aixois de recherche en syntaxe (GARS). Sur base de la transcription orthographique du discours à annoter, nous segmentons le flux de parole en unités, que nous appelons unités de rection (UR). Celles-ci sont ensuite découpées en unités plus petites, les séquences fonctionnelles, selon un découpage décrit par Bilger et Campione (2002). Cette segmentation est largement décrite dans Degand et Simon (2009a). Dans cette section, nous présentons uniquement le découpage en unités rectionnelles, qui seul intervient dans la définition de la BDU.

Une unité rectionnelle s'articule autour d'un noyau, c'est-à-dire un élément recteur. Généralement, l'élément recteur est un verbe tensé, mais il peut être également un constituant averbal, tel qu'un nom, un pronom, un adverbe ou un groupe prépositionnel prédicatif. Ainsi, trois types d'unités de rection peuvent être distingués : (1) les unités de rection verbales (urv), (2) les unités de rection averbales (ura) et (3) les unités de rection elliptiques, qui semblent incomplètes mais qui sont interprétables comme des unités de rection verbale par recours au contexte (ure). Chaque type peut être précisé des mentions "plus» (exemple 4) lorsque l'unité insère un élément non régi par l'unité principale (adjoint, insert ou marqueur du discours) ou « inachevée » (exemple 5) lorsque l'un de ses compléments obligatoires y est absent ou lorsqu'une séquence amorcée est incomplète. 
Dans les conventions de transcription, les unités de rection sont représentées entre crochets droits []; les éléments n'appartenant pas à la rection verbale mais se rapportant tout de même à celle-ci par un lien sémantique ou pragmatique tels que les adjoints, inserts ou marqueurs du discours sont notés entre chevrons $<>$; les séquences fonctionnelles présentes à l'intérieur des unités de rection sont représentées entre parenthèses ( ). Les unités rectionnelles sont de taille variable, allant d'un seul mot à plusieurs dizaines de mots, la longueur ne déterminant pas l'appartenance d'une unité de rection à l'un ou l'autre type.

(1) $\left.\left[(\text { un phonostyle })_{\mathrm{SS}} \text { (va être interprété }\right)_{\mathrm{SV}}<\mathrm{euh}>(\text { par un auditeur })_{\mathrm{SO}}\right]^{\text {urv }}$

(2) [(le quartier Croydon $)_{\mathrm{SN}}$ (sans doute le quartier le plus touché par les émeutes $\left.)_{\mathrm{SN}}\right]^{\text {ura }}$ le chef de l'opposition travailliste Ed Miliband s'exprimera euh lui aussi aujourd'hui pour livrer sa vision des émeutes et des suites à leur donner

(3) L1 vous n'employez pas de dictionnaire de prononciation L2 $\left[(\text { non })_{\mathrm{SAdv}}\right]^{\text {ura }}\left[(\text { aucun dictionnaire de prononciation })_{\mathrm{SN}}\right]^{\text {ure }}$

(4) $\left[(j \text { 'en remercie })_{\mathrm{SV}}<\text { d'ailleurs }>_{\mathrm{md}}<\text { cher Hervé }>_{\mathrm{ad}}<\text { cher Alain }>_{\mathrm{ad}} \text { (tout spécialement }\right)_{\mathrm{SRd}}($ les ministres de la défense qui sont parmi nous ce soir) $\left.)_{\mathrm{SO}}\right]^{\text {urv }+}$

(5) $\left[(\text { on m’a dit })_{\mathrm{SV}}<\mathrm{euh}>\right]^{\text {urv-I }}$

\subsection{Analyse prosodique}

L'annotation prosodique vise la détection de frontières prosodiques majeures et intermédiaires ainsi que l'attribution d'un contour intonatif à chacune de celles-ci. Les seules unités retenues pour la définition des unités de base du discours étant les frontières prosodiques majeures, nous nous centrons uniquement, au sein de cette section, sur les critères menant à leur détection.

Cette détection et l'annotation de frontières est réalisée de manière semi-automatique et se base sur la détection de syllabes proéminentes selon leur hauteur mélodique, leur durée relative ou la durée de la pause subséquente. Une frontière prosodique majeure est identifiée lorsque l'une des contraintes suivantes est identifiée sur la syllabe finale proéminente d'un mot :

- la syllabe finale est suivie par une pause silencieuse d'une durée minimale de $200 \mathrm{~ms}$;

- $\quad$ elle est allongée (trois fois plus longue que la durée moyenne des syllabes précédentes) ;

- elle porte un contour mélodique montant particulièrement saillant (montée ou hauteur relative entre 5 et 10 demi-tons).

Les frontières prosodiques majeures sont notées ///, tandis que les frontières intermédiaires sont notées //. Chaque frontière est suivie par une lettre indiquant le type de contour dont est porteuse la dernière syllabe de l'unité : « $\mathrm{T}$ » désigne un contour terminal (descendant), « $\mathrm{C} »$ un contour continuatif-montant (montée intrasyllabique ou intervalle positif par rapport à la syllabe précédente), « $\mathrm{S}$ » un contour suspens (plat, parfois allongé, sans écart mélodique par rapport à la syllabe qui précède) et « $F$ » un contour de focalisation (mouvement descendant partant d'une cible haute).

\subsection{Types d'unités discursives de base}

Grâce aux découpages syntaxique et prosodique, chacun réalisé de manière autonome, l'identification des BDU peut être réalisée. Celle-ci repose sur la mise en correspondance des deux annotations précédentes, et son principe est simple : chaque fois qu'une frontière d'unité de rection coïncide avec une frontière prosodique majeure, une BDU est identifiée. Cette mise en correspondance donne lieu à quatre types d'unités de base du discours, représentées en figure 1 :

- les BDU congruentes (BDU-C), où une unité de rection syntaxique correspond à une unité prosodique majeure :

$$
\left[(\text { elle courait })_{\mathrm{SV}}(\text { très très vite })_{\mathrm{SRd}}\right]^{\text {urv } \left./ / / /_{(B D U} \mathrm{C}\right)}
$$


- les BDU groupées par l'intonation (BDU-I), où plusieurs unités de rection sont regroupées dans une unité prosodique ;

$<$ ben $>_{\text {md }}\left[(\text { oui })_{\text {SAdv }}\right]^{\text {ura }} / /^{\mathrm{C}}\left[\left(\text { c'est un peu }_{\text {SV }}(\text { la même chose })_{\mathrm{SO}} / /^{\mathrm{T}} \text { (maintenant }\right)_{\mathrm{SRd}}\right]^{\text {urv }} / /^{\mathrm{T}}$

$<$ mais $>_{\text {md }}[\text { (tout est un peu })_{\text {Sv }}$ (la même chose $\left.)_{\text {SO }}\right]^{\text {urv }} / / /^{\mathrm{T}}{ }_{(\text {BDU-I })}$

- les BDU groupées par la syntaxe (BDU-S), où une unité de rection est découpée en plusieurs unités prosodiques majeures ;

[(je n'en ai) $)_{\mathrm{SV}} / / /^{\mathrm{C}}$ (aucune idée) $\left.)_{\mathrm{SO}}\right]^{\text {urv }} / / /^{\mathrm{F}}$ (BDU-S)

- $\quad$ et les BDU régulatives (BDU-R), qui contiennent un élément syntaxiquement non régi (tel qu'un connecteur, un adverbe de phrase ou un marqueur du discours) et isolé par une frontière prosodique.

$<$ honnêtement $>_{\text {ag }} / / /_{\text {(BDU-R) }}^{\mathrm{C}}$

$\left[(\text { je crois })_{\mathrm{SV}}(\text { que c'est pour faire bon garçon respectable })_{\mathrm{SO}}\right]^{\mathrm{urv}} / / /^{\mathrm{T}}$

Enfin, une catégorie (BDU-X) regroupe les restes de l'analyse, c'est-à-dire les BDU contenant plusieurs unités de rection et plusieurs unités prosodiques majeures.

\begin{tabular}{|c|c|c|c|c|}
\hline Syntaxe & {[} & [ ] & ] & $<$ \\
\hline Prosodie & [ & {[} & {$\left[\begin{array}{lll}] & {[}\end{array}\right]$} & {[} \\
\hline BDU & {[} & {$\left[\_\right]$} & [ & {[} \\
\hline & congruente & $\begin{array}{l}\text { groupée par } \\
\text { les unités } \\
\text { prosodiques }\end{array}$ & $\begin{array}{l}\text { groupée par } \\
\text { les unités } \\
\text { syntaxiques }\end{array}$ & régulative \\
\hline
\end{tabular}

Figure 1 - Quatre types de BDU

Dans une étude précédente (Degand et Simon 2009b), nous avons constaté que ces quatre types de BDU remplissent différentes fonctions discursives et se répartissent différemment selon le genre en jeu, parmi quatre genres étudiés. Dans cette contribution, nous souhaitons corroborer cette étude à plus grande échelle, sur notre corpus LOCAS-F que nous présentons dans la section suivante.

Notre précédente étude a également pu montrer que les BDU remplissent une fonction cognitive dans la planification et l'interprétation du discours. Dans ce cadre, les BDU régulatives constituent un cas intéressant : étant constituées uniquement d'un élément syntaxiquement non régi et isolé par une frontière prosodique, elles se caractérisent par l'absence de contenu sémantique prédicatif. Pour Chafe (1994:64), ces unités régulatives peuvent remplir différentes fonctions: "some regulate the development of the discourse [...]. Others have to do with the interaction between the participants [...]. Still others express the speaker's mental process, or judgment of the validity of the information being conveyed 》. Le corpus étudié dans cette précédente étude comprenant la majorité de ses unités régulatives $(68 \%)$ en périphérie gauche de l'énoncé, nous pensons que cette position joue un rôle stratégique dans la structuration du discours. Ce lieu où le message prend forme constitue en effet le point d'ancrage du message, où le contenu du discours peut être relié de manière cohérente aux contenus précédents (Virtanen, 2004 : 8081). Nous pensons que les éléments situés en périphérie gauche des BDU sont, par conséquent, susceptibles de jouer un rôle dans l'organisation informationnelle du discours. Nous revenons sur ces points au sein de la section d'analyse de cette contribution, après avoir présenté notre corpus de travail dans la section suivante.

\section{LOCAS-F : un corpus oral multi-genres annoté}

Le corpus LOCAS-F est constitué de 42 échantillons sonores, chacun d'une durée de 3 à 5 minutes environ et comprend, en tout, 48 locuteurs différents représentant principalement des variétés du français de Belgique et de France. Ces échantillons sont répartis en 12 "genres » ou activités de communication orale différentes (nous suivons ici la conception du genre comme une pratique sociale, telle que préconisée par Miller 1984 ou Rastier 1989). 
Pour caractériser ces différentes activités de parole et rendre les données comparables entre elles, nous avons catégorisé chaque échantillon selon plusieurs traits situationnels du modèle conceptionnel de Koch et Oesterreicher (2001), qui permettent de « catégoriser le comportement communicatif des interlocuteurs par rapport aux déterminants situationnels et contextuels » (2001:586). En regard de l'étude de Simon et al. (2010), où les auteurs déterminent quelles sont les dimensions pertinentes pour refléter la variation stylistique d'échantillons de parole et définir des phonogenres, nous retenons trois traits situationnels susceptibles d'influencer, selon nous, la manière dont le discours est structuré :

- le degré d'interactivité entre les participants : 0 caractérise un discours non interactif ; 1 un discours semi-interactif, où la liberté d'interrompre est restreinte ; 2 un discours interactif, où la parole est librement distribuée ;

- le degré de préparation du discours : 0 caractérise un discours non préparé, spontané ; 1 un discours semi-préparé, dont le sujet est connu par le participant; 2 un discours totalement préparé/lu ;

- le caractère médiatique du discours : 0 caractérise des données non médiatiques ; 1 des données secondairement médiatiques, où l'activité implique plusieurs rôles communicatifs et où les participants ne s'adressent pas directement aux auditeurs/spectateurs; 2 des données médiatiques, exclusivement produites pour être diffusées.

Le tableau 1 ci-dessous réunit chaque genre de notre corpus, décrits selon ces trois critères situationnels. Dans le tableau suivant (2, ci-dessous), nous décrivons le corpus en termes de nombre de mots, d'unités syntaxiques, d'unités prosodiques et de BDU. Nous pouvons déjà constater que la proportion d'unités varie selon le genre en présence : l'interview libre et la narration conversationnelle, par exemple, comprennent davantage d'unités syntaxiques que d'unités prosodiques, indiquant une probable fréquence plus élevée de BDU groupées par la prosodie. A l'inverse, la conférence scientifique, le discours académique et l'homélie, notamment, comptent davantage d'unités prosodiques que d'unités syntaxiques, indiquant très probablement une plus grande proportion de BDU groupées par la syntaxe. Nous vérifions ces intuitions au sein de la section 4.1., où nous étudions la distribution des différents types de BDU au travers des différents genres de notre corpus.

Tableau 1. - Description générale du corpus

\begin{tabular}{|l|l|l|l|l|}
\hline Genre & $\begin{array}{l}\text { Durée } \\
\text { (min:secondes) }\end{array}$ & $\begin{array}{l}\text { Degré } \\
\text { d'interactivité }\end{array}$ & $\begin{array}{l}\text { Degré } \\
\text { préparation }\end{array}$ & $\begin{array}{l}\text { Caractère } \\
\text { médiatique }\end{array}$ \\
\hline $\begin{array}{l}\text { Conférence } \\
\text { scientifique }\end{array}$ & $16: 44$ & 0 & 2 & 0 \\
\hline Débat & $19: 19$ & 2 & 1 & 1 \\
\hline $\begin{array}{l}\text { Discours } \\
\text { académique }\end{array}$ & $15: 18$ & 0 & 2 & 1 \\
\hline Discours politique & $20: 26$ & 0 & 2 & 2 \\
\hline Homélie & $13: 23$ & 0 & 2 & 2 \\
\hline Enquête & $16: 04$ & 1 & 1 & 2 \\
\hline Interview libre & $15: 33$ & 2 & 0 & 1 \\
\hline Interview radio & $20: 29$ & 1 & 1 & 1 \\
\hline Journal parlé & $14: 45$ & 0 & 2 & 2 \\
\hline Lecture & $15: 18$ & 0 & 2 & 1 \\
\hline $\begin{array}{l}\text { Narration } \\
\text { conversationnelle }\end{array}$ & $10: 22$ & 0 & 0 & 0 \\
\hline Création radio & $13: 24$ & 0 & 1 & 2 \\
\hline Total & $3: 11: 05$ & & & \\
\hline & & & & \\
\hline
\end{tabular}


Tableau 2 - Description générale du corpus

\begin{tabular}{|l|l|l|l|l|l|}
\hline Genre & $\begin{array}{l}\text { Nombre } \\
\text { d'unités } \\
\text { syntaxiques }\end{array}$ & $\begin{array}{l}\text { Nombre } \\
\text { d'unités } \\
\text { prosodiques }\end{array}$ & $\begin{array}{l}\text { Nombre } \\
\text { de BDU }\end{array}$ & $\begin{array}{l}\text { Nombre de } \\
\text { mots }\end{array}$ & $\begin{array}{l}\text { Moyenne } \\
\text { mots/bdu }\end{array}$ \\
\hline $\begin{array}{l}\text { Conférence } \\
\text { scientifique }\end{array}$ & 140 & 320 & 112 & 3123 & 27,88 \\
\hline Débat & 535 & 528 & 362 & 4790 & 13,23 \\
\hline $\begin{array}{l}\text { Discours } \\
\text { académique }\end{array}$ & 100 & 401 & 125 & 2508 & 20,06 \\
\hline Discours politique & 194 & 475 & 194 & 3300 & 17,01 \\
\hline Homélie & 136 & 343 & 150 & 1902 & 12,68 \\
\hline Enquête & 292 & 334 & 221 & 2575 & 11,65 \\
\hline Interview libre & 500 & 476 & 370 & 3559 & 9,62 \\
\hline Interview radio & 358 & 474 & 258 & 4288 & 16,62 \\
\hline Journal parlé & 162 & 206 & 124 & 2938 & 23,69 \\
\hline Lecture & 176 & 414 & 184 & 3157 & 17,16 \\
\hline $\begin{array}{l}\text { Narration } \\
\text { conversationnelle }\end{array}$ & 264 & 133 & 117 & 2320 & 19,83 \\
\hline Création radio & 168 & 277 & 131 & 2452 & 18,72 \\
\hline Total & $\mathbf{3 0 3 5}$ & $\mathbf{4 3 8 1}$ & $\mathbf{2 3 4 8}$ & $\mathbf{3 6 9 1 2}$ & $\mathbf{1 7 , 3 5}$ \\
\hline & & & & &
\end{tabular}

\section{Analyses}

\subsection{Distribution des BDU}

La figure 2 résume la distribution des 2348 BDU de notre corpus à travers les 12 activités de communication qui le constituent. Nous pouvons y voir que les BDU ne se distribuent pas de manière égale selon le genre en jeu. Une statistique de Chi-carré appliquée sur nos données confirme que cette différence de distribution est significative $\left(\chi^{2}=602,84 ; \mathrm{dl}=44 ; p<0,001\right)$.

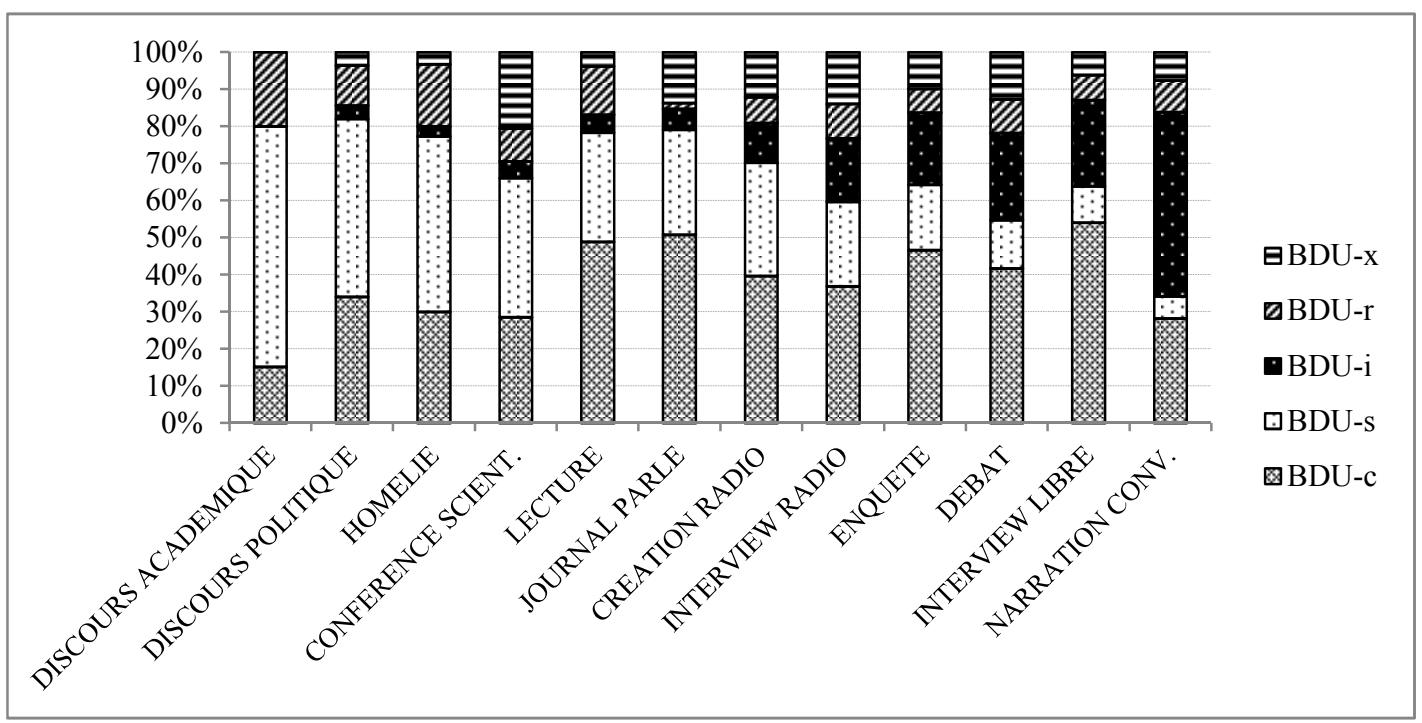

Figure 2 - Distribution des BDU à travers les différents genres du corpus LOCAS-F 
Sur la base du calcul des résidus de la statistique de Chi-carré, nous constatons que trois genres de discours se distinguent des autres par une proportion significativement élevée de BDU groupées par la syntaxe. Il s'agit des discours académiques, des discours politiques et des homélies. Ces trois genres sont tous de type non interactif, préparé et, pour deux d'entre-eux, de type médiatisé (la diffusion étant limitée pour le discours académique). Une telle proportion de BDU-s indique un style plutôt didactique ou emphatique, où la parole est comme «sursegmentée » intonativement et est d'ailleurs typique des genres où le degré de préparation est élevé (Degand et Simon 2011).

À l'inverse, les genres caractérisés par un plus grand degré de spontanéité, tels que la narration conversationnelle et l'interview libre, se distinguent des autres par une proportion significativement élevée de BDU groupées par l'intonation. L'effet qu'une telle proportion de BDU-i produit est que la parole y est davantage délivrée en grands «paquets informatifs ». Selon nous, un tel effet correspond à une stratégie du discours qui permettrait de marquer des liens de cohérence entre plusieurs unités syntaxiques, sans pour autant marquer ces liens de manière explicite.

Les autres types de BDU se distribuent davantage de manière homogène au sein du corpus, à l'exception des interviews libres et des discours académiques, pour lesquels le calcul des résidus présente un résultat significatif pour, respectivement, la proportion de BDU congruentes (indiquant une stratégie discursive impliquant une présentation de l'information de manière neutre) et la proportion de BDU régulatives (remplissant a priori des fonctions plutôt textuelles, de relations de discours et de gestion des topiques).

Ces différences de distribution nous confirment que la BDU est une unité pertinente pour l'analyse des discours oraux. Afin d'étayer ce propos et de confirmer l'assertion selon laquelle la complétude syntaxique et la complétude prosodique ne suffisent pas pour délimiter des unités dans le flux de parole (cf. supra), il est intéressant de comparer les statistiques de Chi-Carré obtenues pour les distributions des BDU avec celles des unités syntaxiques (unités de rection) et des unités prosodiques (contours des frontières intonatives majeures) de notre corpus. La valeur de Chi-Carré étant influencée par le nombre d'effectifs, nous avons calculé le $\mathrm{V}$ de Cramer relatif à chaque Chi-carré obtenu. Cette mesure d'association permet de comparer des valeurs de Chi-Carré entre elles et de déterminer la force de la corrélation entre les deux variables testées. La valeur obtenue est comprise entre 0 (aucune corrélation) et 1 (corrélation parfaite). Le score obtenu pour l'association entre les genres et les types de BDU $\quad(V=$ $0,25)$ est environ 1,5 fois plus élevé que celui obtenu pour la distribution des unités de rection $\quad(V=$ $0,18)$ et des unités prosodiques $(V=0,17)$ à travers les genres. Malgré le caractère peu élevé des valeurs obtenues, cela confirme que le découpage en unités discursives de base explique davantage la variance observée sur l'ensemble des données à travers les genres que le découpage en unités syntaxiques ou en unités prosodiques.

\subsection{Distribution des éléments en périphérie gauche (EPG)}

\subsubsection{Définition des EPG}

À l'instar de l'identification des BDU réalisée grâce à la combinaison de marques syntaxiques et prosodiques, nous identifions les périphéries gauches par des critères syntaxiques et prosodiques : un élément peut être considéré comme périphérique dès lors qu'il est isolé par une frontière syntaxique (élément non régi) ou par une frontière prosodique (majeure), ou encore par une combinaison de ces deux marques. Lorsque l'initiale de la BDU est syntaxiquement et prosodiquement intégrée au reste de la séquence, nous l'étiquetons comme un simple élément initial. Ainsi, notre approche ne se limite pas à l'étude d'une seule position ou fonction (cf. Virtanen 2004 et Charolles et al. 2005 qui traitent isolément les adverbes de temps, de lieu et de manière en position initiale), ni à une liste de catégories syntaxiques (cf. Apothéloz et al. 2009, qui traitent uniquement de nombreuses formes de dislocation syntaxique).

Quatre types de périphéries gauches peuvent être distingués (Degand, Simon, Tanguy et Van Damme, à paraître). Nous les détaillons et exemplifions dans le tableau 3. 
Tableau 3. - Différentes configurations des éléments initiaux à gauche des BDU.

\begin{tabular}{|c|c|c|}
\hline Nom & Description & Exemple \\
\hline$E I$ & $\begin{array}{l}\text { Elément initial, } \\
\text { prosodiquement et } \\
\text { syntaxiquement intégré } \\
\text { à la BDU. }\end{array}$ & $\begin{array}{l}{\left[(\text { il est })_{\mathrm{Sv}}(\text { incontestablement })_{\mathrm{SRd}} / / \text { (un très grand poète }\right.} \\
\left./ / \text { avant tout })_{\mathrm{SO}}\right]^{\mathrm{urv}}\end{array}$ \\
\hline$E P G-S$ & $\begin{array}{l}\text { Elément en périphérie } \\
\text { gauche isolé par une } \\
\text { frontière syntaxique. }\end{array}$ & $\begin{array}{l}<\text { de toute façon }>_{\text {ag }} / / \mathrm{C}\left[\left(\text { c'est }^{\prime} \text { plus facile }\right)_{\mathrm{SV}}\right]^{\mathrm{urv}} / / \mathrm{T} \\
\left.[\text { (ouais })_{\mathrm{SAdv}}\right]^{\text {ura }} / / /\end{array}$ \\
\hline$E P G-I$ & $\begin{array}{l}\text { Elément en périphérie } \\
\text { gauche isolé par une } \\
\text { frontière prosodique. }\end{array}$ & 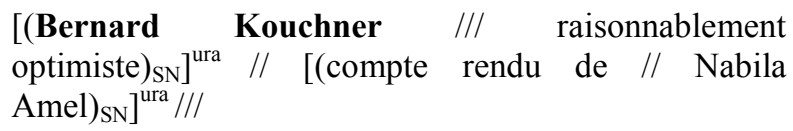 \\
\hline$E P G$ & $\begin{array}{l}\text { Elément en périphérie } \\
\text { gauche isolé par une } \\
\text { frontière syntaxique et } \\
\text { prosodique (correspond } \\
\text { à une BDU-R). }\end{array}$ & $\begin{array}{l}<\text { pour autant }>_{\text {md }} / / /\left[\left(l^{\prime} \text { Académie }\right)_{S S}(\text { n'est pas })_{S v} / / /\right. \\
\text { (l'unique // horizon de l'UCL en Communauté } \\
\left.\text { Française })_{\mathrm{SO}}\right]^{\text {urv } / / /}\end{array}$ \\
\hline
\end{tabular}

Dans les deux sections suivantes, nous entreprenons l'analyse de la distribution des éléments en périphérie gauche au sein du corpus LOCAS-F. L'hypothèse que nous poursuivons à travers cette analyse concerne la variation des EPG à travers les genres : nous pensons que les formes et les fonctions discursives des périphéries gauches varient avec la situation de production des discours, de la même manière que les BDU varient en fonction du discours en jeu. La position initiale constituant une position stratégique pour le développement du discours (Ho-Dac, 2007), nous pensons que sa variation formelle et fonctionnelle à travers les genres peut montrer que les éléments en périphérie gauche constituent des expressions au service de la cohésion discursive, et qu'ils participent d'une stratégie de discours.

\subsubsection{Distribution formelle des EPG}

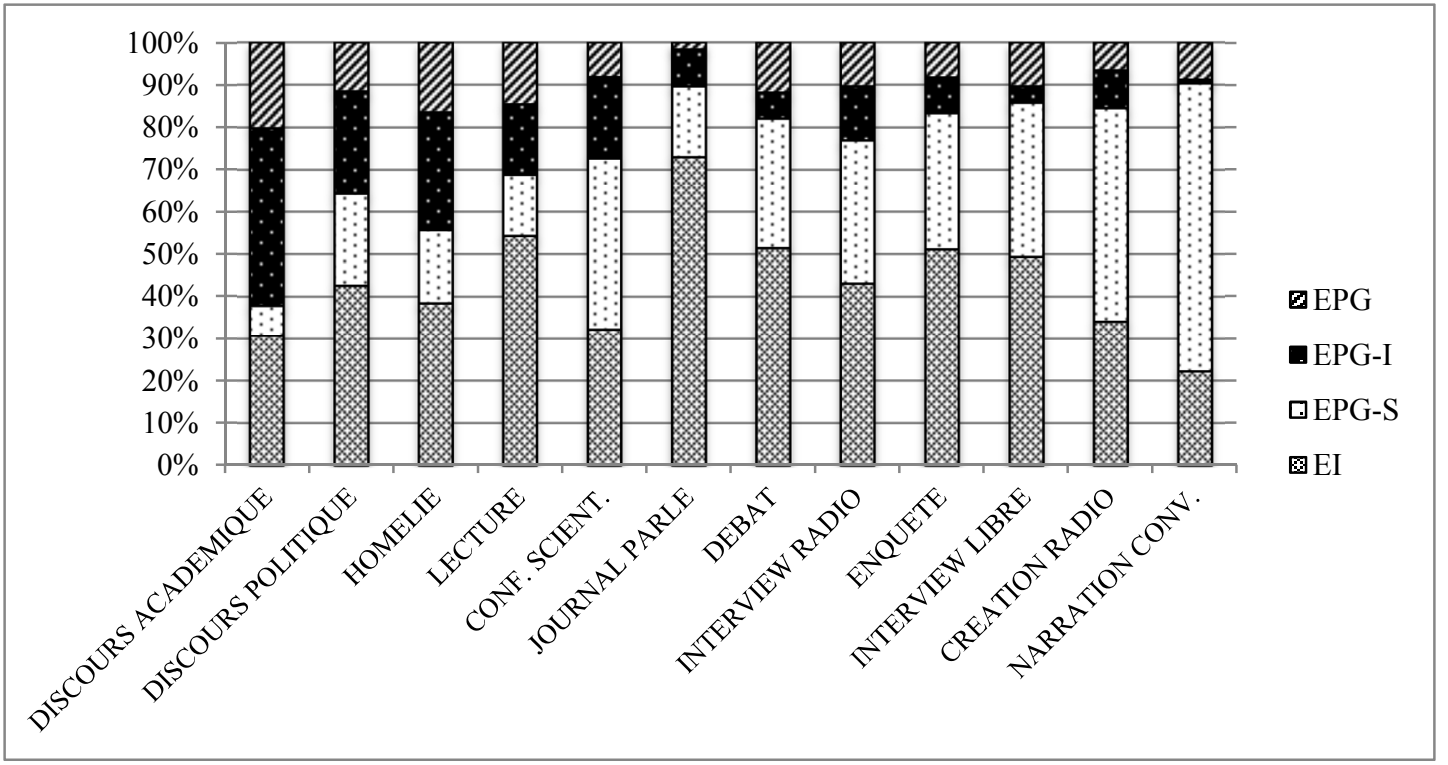

Figure 3. - Distribution des éléments à gauche à travers les genres 
La figure 3 ci-dessus résume la répartition des éléments situés à gauche des BDU (périphériques ou intégrés) au sein des différents genres du corpus LOCAS- $\mathrm{F}^{4}$. Nous pouvons constater que ces éléments, à l'instar des BDU, ne se distribuent pas au hasard à travers les genres. Le Chi-Carré calculé sur cette distribution est significatif $\left(\chi^{2}=394,08 ; \mathrm{dl}=33 ; p<0,001\right)$, et le $\mathrm{V}$ de Cramer associé est aussi élevé que celui calculé pour la distribution de $\operatorname{BDU}(V=0,25)$.

Cette figure nous permet de constater que la distribution des EPG-I et des EPG-S varie fortement selon le genre : dans la partie gauche du graphique, trois genres se distinguent par un nombre significativement élevé d'EPG-I ainsi qu'un nombre significativement réduit d'EPG-S. Il s'agit du discours politique, du discours académique et de l'homélie. Ces trois discours se distinguant déjà des autres par leur nombre élevé de BDU groupées par la syntaxe, il semble qu'une telle proportion d'éléments en périphérie gauche isolés par la prosodie soit le reflet la «sursegmentation » prosodique dont ils font preuve.

Dans la partie droite du graphique, la tendance s'inverse : les narrations conversationnelles et les créations radio présentent un nombre significativement élevé d'EPG isolés par une frontière syntaxique. À ce stade de l'étude, nous ne possédons pas encore d'explication satisfaisante pour justifier ce cas. Nous pouvons remarquer que ces deux genres sont tous deux plutôt spontanés, et il semblerait que ce facteur influence la proportion d'EPG-S et d'EPG-I au sein du genre représenté. La figure 4 confirme que le paramètre situationnel de degré de préparation du discours et la distribution des EPG ne sont pas indépendants $\left(\chi^{2}=\right.$ 181,$21 ; \mathrm{dl}=6 ; p<0,0001$; résidus significatifs pour la distribution des EPG-I et des EPG-S à travers les trois catégories).

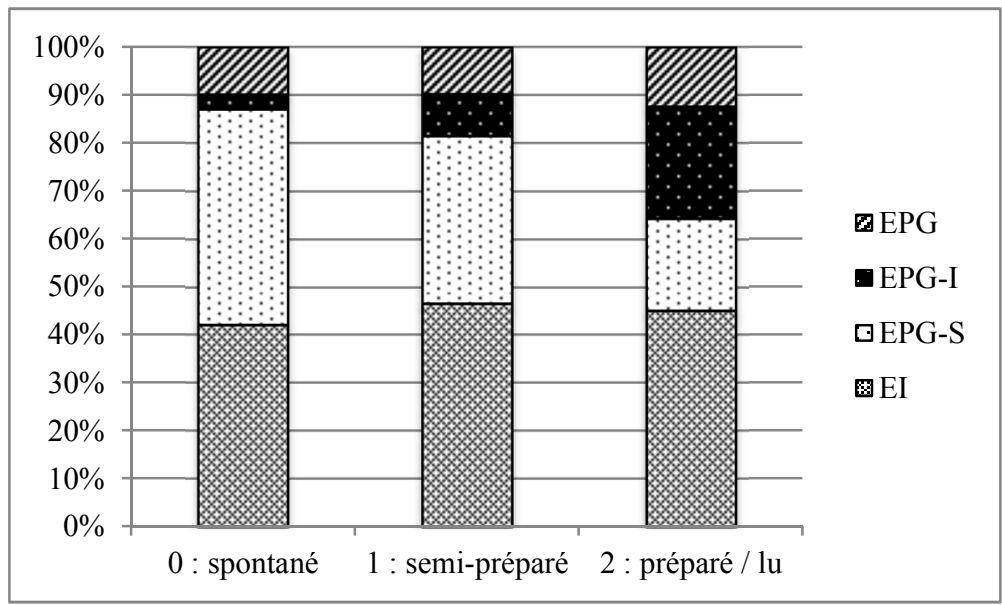

Figure 4. - Distribution des éléments à gauche selon le degré de préparation du discours

\subsubsection{Distribution fonctionnelle des EPG}

Pour comparer les fonctions que peuvent revêtir les éléments en périphérie gauche et étudier leur distribution selon la situation de communication, nous avons étiqueté un échantillon du corpus LOCAS-F. Celui-ci comprend deux enregistrements très différents l'un de l'autre quant à leurs paramètres situationnels : l'extrait de conférence scientifique, d'une part, est un discours monologique se situant du côté de la distance communicative, tandis que l'extrait de l'interview libre, d'autre part, est dialogique et est proche de l'immédiat communicatif. Les catégories choisies pour l'étiquetage fonctionnel sont inspirées de Chafe (1994:64) et permettent de distinguer les fonctions textuelle, interactive et cognitive.

La fonction textuelle désigne une relation sémantique introduite par l'élément périphérique entre deux contenus sémantiques du discours. Cette relation sémantique peut être de type logique (exemple 6), marquer la gestion topicale du discours (exemple 7) ou encore être de type métadiscursive, en pointant le discours en développement (exemple 8). 
Tandis que la fonction textuelle est orientée vers le texte, la fonction interactive est orientée vers les participants : l'élément situé en périphérie gauche qui revêt une telle fonction marque en effet soit une interpellation (exemple 9), lorsque le locuteur attire l'attention de l'interlocuteur dans son discours, soit un changement de tour de parole (exemple 10), lorsqu'il signale la prise de parole du locuteur.

Enfin, la fonction cognitive est orientée vers le locuteur lui-même, en marquant sa subjectivité comme le montre l'exemple 11. Notons qu'un EPG peut recevoir plusieurs catégories fonctionnelles, comme dans l'exemple 12. où l'élément périphérique isolé par la syntaxe «parce que parler parler » marque une relation textuelle logique d'une part via la présence de la locution "parce que », et une relation textuelle de gestion topicale d'autre part en introduisant le sujet de «parler ».

(6) $\quad<$ mais $>_{\text {md }}\left[(\text { on ne voyait rien })_{\text {Sv }}\right]^{\text {urv } / / /}$

(7) <ce génie national $>_{\mathrm{ag}} / /\left[(\text { on le trouve })_{\mathrm{SV}} / / \text { (principalement) }\right)_{\mathrm{SRd}} / /$ (dans les traditions /// qui doivent capter // et formuler ce génie national $\left.)_{\mathrm{so}}\right]^{\text {urv } / / /}$

(8) [(ce sera le premier point // de de mon exposé qui en port/ comportera

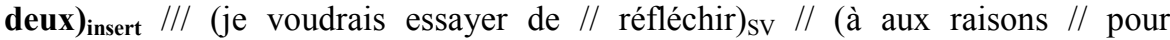
lesquelles /// le facteur linguistique // semble jouer /// une espèce de de de rôle primordial // dans la constitution des identités) $\left.)_{\mathrm{so}}\right]^{\text {urve }} / / / /$

(9) $<$ \{alors, là $\}>_{\text {md }}<$ vous $>_{\text {ag }} / /$ [(vous êtes plus // doux // vous êtes plus doux // que moi) $\left.)_{\mathrm{sv}}\right]^{\text {urv }} / / /$

(10) <en fait $>_{\mathbf{m d}} / /\left[(j \text { 'étais) })_{\mathrm{SV}}\right.$ (un enfant /// très très malheureux // euh très renfermé $\{$ et, -$\} / / \mathrm{S}$ tout ça $\left.)_{\mathrm{SO}}\right]^{\text {urv }} / / /$

(11) $<$ ben $>_{\text {md }}\left[(\text { la Grande Bouffe })_{\mathrm{SN}}\right]^{\text {ura } / / /}$

(12) $<$ parce que parler //C parler $>_{\text {ad }} / /\left[(\text { vous savez })_{s v}\right]^{\text {urv }}<$ moi $>_{\text {ag }}[$ (j'ai pas $/ / /$ j'ai

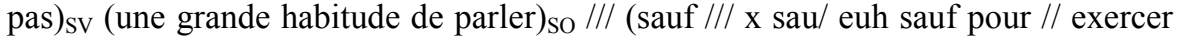
mon métier) $\left.)_{S R d}\right]^{\text {urv }} / / /$

Le tableau 4 ci-dessous résume la répartition des différentes fonctions des EPG étudiés pour cette contribution. Nous pouvons y lire que la majorité des EPG participe à la progression textuelle grâce à de nombreuses relations logiques. La conférence scientifique présente également de nombreuses EPG revêtant une fonction de gestion topicale, à l'inverse de l'interview qui en présente peu. Il est particulièrement frappant de constater que les EPG de la conférence scientifique remplissent exclusivement des fonctions textuelles, qui indiquent des stratégies discursives de planification et d'organisation du discours. L'interview, par contre, comprend quelques éléments périphériques de type interactif ou cognitif. Grâce à la présence de telles fonctions au sein de ce genre, la différence entre la distance communicative et l'immédiat communicatif se remarque aisément : il s'agit en effet de mettre en œuvre, à travers ces fonctions interactive et cognitive, des stratégies de management et de négociation du flux de parole conversationnelle, et de marquer la subjectivité du locuteur.

Table 4. - Fonctions discursives des EPG à travers deux échantillons

\begin{tabular}{|l|l|l|l|l|l|l|}
\cline { 2 - 7 } \multicolumn{1}{c|}{} & \multicolumn{3}{c|}{ Textuelle } & \multicolumn{2}{c|}{ Interactive } & Cognitive \\
\cline { 2 - 7 } & $\begin{array}{l}\text { Relation } \\
\text { Logique }\end{array}$ & $\begin{array}{l}\text { Gestion } \\
\text { topicale }\end{array}$ & $\begin{array}{l}\text { Méta- } \\
\text { discours }\end{array}$ & Turn-taking & $\begin{array}{l}\text { Interpel- } \\
\text { lation }\end{array}$ & \\
\hline Conférence & $11(40,7 \%)$ & $12(44,4 \%)$ & $4(14,8 \%)$ & $0(0 \%)$ & $0(0 \%)$ & $0(0 \%)$ \\
\hline Interview & $20(52,6 \%)$ & $3(7,9 \%)$ & $0(0 \%)$ & $6(15,8 \%)$ & $2(5,3 \%)$ & $7(18,4 \%)$ \\
\hline
\end{tabular}




\section{Conclusion}

L'objectif de cette contribution était de présenter le corpus multi-genres LOCAS-F ainsi que son unité principale de segmentation, la BDU. Nous défendons l'idée que syntaxe et prosodie fournissent chacune des signaux pour la délimitation d'unités dans le flux de parole, et que leur combinaison en unités discursives de base mène au niveau proprement discursif. En comparant la distribution des BDU avec celle des unités syntaxiques et prosodiques de notre corpus, nous avons pu montrer en quoi cette unité est pertinente pour la description des discours oraux.

Nous avons en effet pu confirmer que la distribution des Unités Discursives de Base ainsi que celle de leurs périphéries gauches varient selon l'activité de communication qui est en jeu. Ainsi, le type de BDU et le type d'EPG peuvent constituer des critères discriminants pour la classification et la caractérisation des genres discursifs.

Nous avons vu en effet qu'en fonction de sa distribution en BDU et en EPG, un genre reflètera davantage l'une ou l'autre stratégie discursive. Plus particulièrement, les genres représentatifs de la distance communicative, avec un degré de préparation élevé et peu d'interaction entre locuteurs, présentent davantage de BDU regroupées par la syntaxe et de périphéries gauches isolées par la prosodie. Une telle distribution indique une stratégie discursive plutôt didactique ou emphatique, dont les EPG marqueraient, a priori, des relations textuelles principalement. A l'inverse, les genres représentatifs de l'immédiat communicatif présentent une répartition qui privilégie les BDU groupées par l'intonation et les EPG-s, tels que les marqueurs discursifs, qui fonctionnent davantage comme marqueurs interactifs et cognitifs.

Enfin, nos premiers résultats sur la distribution des fonctions des EPG sont encourageants : le profil fonctionnel des périphéries gauches d'un discours semble pertinent pour la caractérisation des genres discursifs, en particulier sur l'axe opposant la distance communicative et l'immédiat communicatif. Pour le moment, nos données sont cependant trop peu nombreuses pour tirer des conclusions statistiques de ces observations. Nos travaux futurs porteront sur l'élargissement de cette annotation fonctionnelle à l'ensemble du corpus LOCAS-F : ainsi nous pourrons établir une typologie fonctionnelle et paradigmatique des périphéries gauches et étudier leur rôle dans la négociation de la structure discursive.

\section{Bibliographie}

APOTHÉLOZ, D. COMBETTES, B. et NEVEU, F. (éd). (2009). Les linguistiques du détachement. Berne : Peter Lang.

ASHER, N. et LASCARIDES, A. (2003). Logics of Conversation. Cambridge : Cambridge University Press.

BILGER, M. et CAMPIONE, E. (2002). Propositions pour un étiquetage en 'séquences fonctionnelles'. Recherches sur le français parlé, 17, pages 117-136.

Blanche-BenVeniste, C., BILger, M., ROUget, Ch., VAn Den EYNDE, K. et MERTENS, P. (1990). Le français parlé : études grammaticales. Paris : Editions du CNRS.

CHAFE, W.L. (1994). Discourse, consciousness, and time. Chicago : University of Chicago Press.

Charolles, M., Le Draoulec, A., Pery-Woodley, M-P. et SARDA, L. (2005). Temporal and spatial dimensions of discourse organisation. French Language Studies, 15, pages 115-130.

DEGAND, L. et SIMON, A-C. (2008). Minimal Discourse Units in Spoken French: Un-covering Genrebound Segmentation Strategies. In RAMM, W. et FABRICIUS-HANSEN (eds) : Linearisation and Segmentation in Discourse, Lysebu, Oslo: Department of Literature, Area Studies and European Languages, University of Oslo, pages 31-42.

DEGAND, L. et SIMON, A-C. (2009a). On identifying basic discourse units in speech: theoretical and empirical issues. In Discours 4 [En ligne]. URL http://discours.revues.org/index.html. 
DEGAND, L. et SIMON, A-C. (2009b). Mapping prosody and syntax as discourse strategies: How Basic Discourse Units vary across genres. In WICHMANN, A., BARTH-WEINGARTEN, D. et DEHÉ, N. (eds) : Where prosody meets pragmatics : research at the interface, Studies in Pragmatics. Bingley: Emerald, pages 79-105.

DEGAND, L. et SIMON, A-C. (2011). L'analyse en unités discursives de base : pourquoi et comment ?. In Langue française (2011), pages 45-59.

DEGAND, L., SIMON, A-C, TANGUY, N. et VAN DAMME, TH. (à par.). Initiating a discourse unit in spoken French: Prosodic and syntactic features of the left periphery. In PONS BORDERIA, S. (ed) : Theoretical Models of Discourse Segmentation, John Benjamins.

HALFORD, B. K. (1996). Talk Units : The Structure of Spoken Canadian English. Tübingen : Narr.

HALLIDAY, M.A.K. (1994). An Introduction to Functional Grammar. London : Arnold.

HO-DAC, L-M. (2007). La position initiale dans l'organisation dans l'organisation du discours: une exploration en corpus. Thèse de doctorat, Toulouse : Université Toulouse le Mirail.

KOCH, P. et OESTERREICHER, W. (2001). Langage parlé et langage écrit. In HOLTUS, G., METZELTIN, M. et SCHMITT, C. (éd), Lexikon der romanistischen Linguistik, 1/2, pages 584-627.

LACHERET-DUJOUR, A. et VICTORRI, B. (2002). La période intonative comme unité d'analyse pour l'étude du français parlé : modélisation prosodique et enjeux linguistiques. Verbum, 24, pages 55-72.

MILLER, C-R. (1984). Genre as social action. Quarterly journal of speech, 70(2), pages 151-167.

RASTIER, F. (1989). Sens et Textualité. Paris : Hachette.

ROULET E. (1991). À propos des niveaux de l'analyse linguistique. In LIVER, R., WERLEN, I. et WUNDERLI, P. (éd.), Sprachtheorie und Theorie der Sprachwissenschaft, Tübingen : Gunter Narr Verlag, pages 221-230.

ROULET, E., FILLIETTAZ, L., GROBET, A. en collaboration avec BURGER, M. (2001). Un modèle et un instrument d'analyse de l'organisation du discours. Bern : Peter Lang.

ROULET, E. 2002. Le problème de la définition des unités à la frontière entre le syntaxique et le textuel. Verbum, 24, pages161-178.

SACKS, H., SCHEGLOFF, E. et JEFFERSON, G. (1974). A simplest systematics for the organization of turn-taking in conversation. Language 50/4, pages 696-735.

SELTING, M. (2000). The construction of units in conversational talk. Language in Society, 29, pages 477-517.

SIMON, A-C., AUCHLIN, A., AVANZI, M. et GOLDMAN, J-Ph. (2010). Les phonostyles. Une description prosodique des styles de parole en français. In ABECASSIS, M. et LEDEGEN, G. (éd). Les voix des français. En parlant, en écrivant, vol. 2, Berne : Peter Lang, pages 71-88.

VIRTANEN, T. (2004). Point of departure : Cognitive aspects of sentence-initial adverbials. In VIRTANEN, T. (éd.), Approaches to cognition through text and discourse, Berlin/New York : Mouton de Gruyter, pages 79-97. 


\footnotetext{
${ }^{1}$ Nous remercions Thomas Van Damme (Universiteit Gent) et Noalig Tanguy (LaTTiCe, Université Paris 3 Sorbonne Nouvelle) pour leur collaboration à la constitution et à l'annotation du corpus LOCAS-F.

${ }^{2}$ Interview de type informel, réalisée dans le cadre de l'émission belge "Hep Taxi!".

${ }^{3}$ Temps d'un 45 tours devant un micro ouvert, cfr. www.arteradio.com, classé sous "chroniques - art".

${ }^{4}$ Notre corpus comprend 1178 éléments étiquetés comme périphériques et 962 éléments étiquetés comme initiaux. Pour le moment, nous avons écarté de l'analyse les BDU constituées d'une seule séquence et d'une seule frontière prosodique majeure, car elles ne contiennent ni élément initial, ni élément périphérique.
} 